\title{
Evaluation of Maternal and Perinatal Outcome in Pregnancy with Jaundice in a Tertiary Care Hospital
}

\author{
Mihir Kumar Sarkar' ${ }^{1}$, Arindam Halder ${ }^{2}$ \\ 1,2 Department of Obstetrics and Gynaecology, Chittaranjan Seva Sadan \\ Shishu Sadan College of Obstetrics and Gynaecology, Kolkata, West Bengal, India.
}

\section{ABSTRACT}

\section{BACKGROUND}

Jaundice in pregnancy contributes to a significant proportion of maternal and perinatal morbidity and mortality in our country. Overall incidence in India is 1 - 4 per 1000 deliveries. There are increased risks of preterm labour, low birth weight babies, meconium-stained liquor, intrauterine fetal death and postpartum haemorrhage. The purpose of this study was to assess the fetomaternal outcome in pregnancy with jaundice in a tertiary level hospital.

\section{METHODS}

This was a two-year retrospective observational study conducted by reviewing the records of jaundice in pregnancy from April 2018 to March 2020.

\section{RESULTS}

Viral hepatitis comes out as the most common cause accounting for $70.37 \%$ of the cases, whereas intrahepatic cholestasis with an incidence of $20.37 \%$ comes out as the second aetiology. Post-partum haemorrhage is depicted as the major maternal complication whereas prematurity and birth asphyxia come out as the major perinatal complications.

\section{CONCLUSIONS}

The present study projects viral hepatitis (Hepatitis A and E) as the major cause of jaundice in pregnancy. Although low maternal mortality has been recorded in the present study, a high perinatal mortality due to low birth weight and birth asphyxia remains to be a matter of major concern.

\section{KEY WORDS}

Jaundice in Pregnancy, Viral Hepatitis, Postpartum Haemorrhage, Prematurity, Birth Asphyxia
Corresponding Author:

Dr. Arindam Halder,

Suravi, Udayan Housing Complex,

Pratapgarh, Narendrapur,

Kolkata-700103, West Bengal, India.

E-mail: halderarindam1978@gmail.com

DOI: $10.14260 / \mathrm{jemds} / 2021 / 632$

How to Cite This Article:

Sarkar MK, Halder A. Evaluation of maternal and perinatal outcome in pregnancy with jaundice in a tertiary care hospital. J Evolution Med Dent Sci 2021;10(36):3099-3102, DOI: 10.14260/jemds/2021/632

Submission 25-03-2021, Peer Review 29-05-2021, Acceptance 05-06-2021, Published 06-09-2021.

Copyright (C) 2021 Mihir Kumar Sarkar et al. This is an open access article distributed under Creative Commons Attribution License [Attribution 4.0 International (CC BY 4.0)] 


\section{BACKGROUND}

Jaundice in pregnancy, although uncommon, poses a significant challenge to the clinician because of the large number of diagnostic possibilities and because of the fact that it carries a substantial risk for the mother and the foetus. Prompt and accurate diagnosis is necessary as multidisciplinary management in a referral centre can improve the maternal and foetal outcome to a great extent. In pregnancy, gall bladder motility decreases and lithogenic index of bile increases resulting in increase of gallstone formation due to hormonal effect. ${ }^{1}$ There is increase in serum alkaline phosphatase level because of placental secretion. Total and free bilirubin is lower in all trimester while conjugated bilirubin level is reduced in second and third trimester. The incidence of abnormal liver test in pregnancy is $3-5 \% .^{2}$

Any increase in these values reflects hepatobiliary disease. Thus, a pregnant patient with abnormal liver test should undergo standard workup as in non-pregnant female which includes proper history taking, clinical examination and serological tests.

In cholestatic disease, the bile secretion is impaired, so increase in bilirubin and alkaline phosphatase level and little rise in aminotransferase level occurs. Jaundice and pruritus are the main complaints but pain in upper abdomen is seen in hyperemesis gravidarum and intrahepatic cholestasis. In hepatocellular disease, occurrence of jaundice is late, epigastric pain with nausea and vomiting are the main complaints with high transaminases level as seen in viral hepatitis and acute fatty liver of pregnancy.3,4

HELLP syndrome is characterized by haemolytic anaemia, elevated liver enzymes and low platelets. It typically presents between 28 and 36 weeks of gestation and $30 \%$ cases occur in first week postpartum. The incidence is $0.1-0.6 \%$ of all pregnancies and $4-12 \%$ of women with preeclampsia. ${ }^{5}$ Acute fatty liver of pregnancy is rare life threating condition. Risk factors are low body mass index and twin pregnancy. It can progress to pancreatitis, renal failure, disseminated intravascular coagulation (DIC), hepatic failure, uncontrolled gastrointestinal and uterine bleeding and death. ${ }^{6}$

\section{METHODS}

This was a retrospective observational study that was conducted in the Dept. of Gynaecology \& Obstetrics, Chittaranjan Seva Sadan, Kolkata from April 2018 to March 2020. All the required data was collected from the record section of CSS.

The aims and objectives of the study are to find out: -

1. The precise aetiology of jaundice in pregnancy.

2. Maternal and perinatal outcome.

\section{Inclusion Criteria}

All cases of clinically detectable jaundice (serum bilirubin $>2$ mg \%) irrespective of age, parity and duration of pregnancy who were admitted in the obstetric ward during the aforesaid period were included in the study. A total of 54 cases were taken in the study.

\section{Exclusion Criteria}

Associated major medical or obstetrical complications such as severe anaemia, diabetes, antepartum haemorrhage (APH) which may adversely affect the prognosis of a particular case, were excluded from the study.

All the selected cases were subjected to a detailed history specially regarding age, parity, symptoms related to jaundice and medications. Past obstetric, medical, and surgical history were recorded.

Thorough general and obstetric examinations were done next. Apart from routine antenatal investigations, the special investigations done in each case were liver functions test (Serum bilirubin, AST, ALT and alkaline phosphatase), Coagulation profile (bleeding time, clotting time, prothrombin time) and hepatitis serology (HBsAg, IgM to HAV, HEV and HCV). Renal function test and 24 hours urinary protein estimation were done in selected group of patients. Ultrasonography (USG) was done in all cases for foetoplacental profile and upper abdominal USG was done to exclude gall bladder stone and extra hepatic obstruction. Pregnancy, labour, post-partum events were recorded. Neonate was observed immediately after birth and a record was kept regarding the first seven days of life.

\section{Statistical Analysis}

Data analysis was carried out using Statistical Package for Social Sciences (SPSS) IBM version 21.0. Descriptive statistics such as mean, standard deviation and range values were computed for quantitative variables. Frequency data across categories were compared using chi - square/Fishers exact test as appropriate.

\section{RESULTS}

Majority of the patients were young primiparas (18 - 30 years - $88.89 \%$ and P0 - $51.85 \%$ ). They were mostly admitted during third trimester i.e. $96.30 \%$ and $75.92 \%$ had mild jaundice at the time of admission.

Regarding the aetiological aspect of jaundice with pregnancy, present study clearly indicates viral hepatitis (HAV, HEV, HBV, HCV) to be the most common cause accounting for $70.37 \%$ of the cases whereas intrahepatic cholestasis with an incidence of $20.37 \%$ comes out as the second aetiology. Preeclampsia and obstructive jaundice due to gall stone are less important causes accounting for $5.56 \%$ and $3.70 \%$ respectively (Table 1 ). As far as labour was concerned, it was spontaneous in onset in $77.77 \%$ of the cases whereas induction was done in $22.22 \%$ of the cases. $53.70 \%$ of the patients delivered at term (37 - 40 weeks); $42.59 \%$ before 37 weeks; only 2 unbooked cases (3.70\%), delivered after 40 weeks as shown in Table 2.

Most of the cases i.e., $83.33 \%$ had normal delivery; lower segment caesarean section (LSCS) was needed in $16.67 \%$ of the cases (Table 3). Major maternal complications as depicted in Table 4 in order of frequency of occurrence were $\mathrm{PPH}$ (27.78\%); hepatic failure and coma (11.11\%); maternal death (5.55 \%); renal and DIC (1.85 \% each). 
The perinatal mortality in the present study was quite high $(27.78 \%)$ and most of the deaths were related to low birth weight, prematurity, and birth asphyxia. The recorded preterm intrauterine fetal death (IUFD), low birth weight, low APGAR (appearance, pulse, grimace, appearance, and respiration) score at birth were $42.59 \%, 11.11 \%, 48.14 \%$ and $33.33 \%$ respectively (Table 10 and Table 5).

\begin{tabular}{|c|c|c|c|}
\hline & No & Total Viral \% & $\%$ \\
\hline \multirow{4}{*}{ Viral Infection } & 12 & \multirow{4}{*}{$70.37 \%$} & $22.22 \%$ \\
\hline & 7 & & $12.96 \%$ \\
\hline & 10 & & $18.25 \%$ \\
\hline & 1 & & $1.85 \%$ \\
\hline \multirow{2}{*}{$\begin{array}{l}\text { Possible viral infection (Viral Status not known) } \\
\text { Intrahepatic cholestasis of pregnancy }\end{array}$} & 8 & \multicolumn{2}{|l|}{$14.82 \%$} \\
\hline & 11 & \multicolumn{2}{|l|}{$20.37 \%$} \\
\hline $\begin{array}{l}\text { Intrahepatic cholestasis of pregnancy } \\
\text { Severe preeclampsia }\end{array}$ & 3 & \multicolumn{2}{|l|}{$5.56 \%$} \\
\hline Obstructive jaundice (due to GB stone) & 2 & \multicolumn{2}{|l|}{$3.70 \%$} \\
\hline \multicolumn{4}{|c|}{ Table 1. Aetiology } \\
\hline
\end{tabular}

\begin{tabular}{|ccc|}
\hline Period of Gestation & No & Percentage \\
$<37$ weeks & 23 & $42.59 \%$ \\
$37-40$ weeks & 29 & $53.70 \%$ \\
$>\quad 40$ weeks & 2 & $3.70 \%$ \\
\hline \multicolumn{3}{|c|}{ Table 2. Time of Delivery } \\
\hline
\end{tabular}

\begin{tabular}{|ccc|}
\hline Mode of Delivery & No & Percentage \\
Normal & 45 & $83.33 \%$ \\
LSCS & 9 & $16.67 \%$ \\
Forceps / Ventouse / Destructive & 0 & 0 \\
\hline \multicolumn{3}{|c|}{ Table 3. Mode of Delivery } \\
\hline
\end{tabular}

\begin{tabular}{|ccc|}
\hline Maternal Complication & No & Percentage \\
PPH & 15 (2 required subtotal hysterectomy) & $27.78 \%$ \\
Hepatic failure \& coma & 6 & $11.11 \%$ \\
Renal failure & 1 & $1.85 \%$ \\
DIC & 1 & $1.85 \%$ \\
Maternal death & 3 & $5.55 \%$ \\
Uneventful recovery & 29 & $53.70 \%$ \\
\hline \multicolumn{2}{|c|}{ Table 4. Major Maternal Complications } \\
\hline
\end{tabular}

\begin{tabular}{|cc|c|}
\hline Perinatal Outcome & No & Percentage \\
\hline Preterm & 23 & $42.59 \%$ \\
IUFD & 6 & $11.11 \%$ \\
LBW (below 2.5 Kg) & 26 & $48.14 \%$ \\
Low APGAR at birth & 18 & $33.33 \%$ \\
(Below 5 Kg) & 4 (out of 7 HBsAg positive & $57.14 \%$ \\
HBsAg positive babies & mothers) & - \\
Neonatal hepatitis & - & $27.78 \%$ \\
\hline Perinatal mortality & 15 & \\
(stillbirth + death in 1st week) & \multicolumn{2}{|c|}{ Table 5. Perinatal Outcome } \\
\hline \multicolumn{2}{|c}{} \\
\hline
\end{tabular}

\section{DISCUSSION}

The very fact that majority of the patients were young primiparas in the present study was purely incidental and no such correlation is found to exist in previous studies. Regarding the aetiological aspect of jaundice, present study projecting viral hepatitis (HAV, HEV, HBV and HCV) to be the most common cause $(70.37 \%)$ is in accordance with a study by Lahiri et al. 1976 who reported a $90 \%$ incidence of viral hepatitis in his series of 290 cases although Griffen et al. ${ }^{8}$ 1969 reported only $42 \%$ incidence of viral hepatitis in his series. There is an uneven worldwide incidence of intrahepatic cholestasis varying from 1 in 1000 to 1 in 10000 deliveries. ${ }^{1}$ The commonest viral agent causing hepatitis, in the present series is HAV (22.22 \%) followed closely by HEV (18.51\%) and HBV (12.96\%). However, viral status of 8 patients (14.82\%) who clinically appeared to be of infective aetiology was unknown due to non-availability of reports and that might have altered the statistic to some extent. In a similar study from Zagreb, ${ }^{9}$ the incidence of HAV (18.4\%), HBV (57.9 \%), Non-A Non-B (23.7 \%) were recorded. However, most of the epidemics of viral hepatitis in India and the majority of the sporadic cases of hepatitis in pregnancy are reported to be due to NANB type of virus by different authors. (Nayak, 1985; Chadha et al. 1987). ${ }^{10}$

As already described, labour was spontaneous in onset in $77.77 \%$ of the cases whereas induction was done in $22.22 \%$ of the cases. The main indications for induction were intrahepatic cholestatic cases after 37 weeks (8 cases), severe preeclampsia with jaundice ( 2 cases) and viral hepatitis with post-dated pregnancy ( 2 cases). There is accumulating evidence by different authors that induction after 37 completed weeks reduces perinatal mortality and morbidity in intrahepatic cholestasis of pregnancy. ${ }^{6}$

While $83.33 \%$ of women had normal delivery, ISCS was required in $16.67 \%$ cases and the indications were cephalopelvic disproportion (CPD) in 2 cases, non-progress of labour in 2 cases, post CS pregnancy with leaking membranes in 2 cases and fetal distress in rest 3 . It can be emphasized that all the indications for LSCS were obstetrical and jaundice per se is not an indication for LSCS.

Of the maternal complications, PPH remained the most important one occurring in 15 cases $(27.78 \%)$ of which 8 needed blood transfusion and 2 needed subtotal hysterectomy. Hepatic failure with coma developed in 6 patients (11.11\%) in the present series and all those cases interestingly developed hepatic encephalopathy soon after normal delivery. One patient out of these 6 , had renal failure and another one had DIC and both succumbed. 4 patients of hepatic failure could be saved by intensive multidisciplinary care. Acute liver failure was reported in $22.2 \%$ of the cases in the study from Kashmir. ${ }^{11}$

Maternal death occurred in 3 cases (5.55 \%) as already mentioned. One of them had hepatic failure with renal failure; the second had hepatic failure with DIC; their patient died due to intractable PPH despite medical and surgical measures. It is worthy to note that 29 cases $(53.70 \%)$ had uneventful recovery. However, earlier studies showed a high maternal mortality of (20 - $39 \%$ ) (Khuroo, 1980; Tandon et al. 1982),12,5 and most of the deaths in their series were due to hepatic failure and $\mathrm{PPH}$.

The perinatal mortality in the present study was quite high $(27.78 \%)$ and most of the deaths were related to low birth weight, prematurity and birth asphyxia. Available reports show a much lower perinatal mortality [ $10-15 \%$ by Reid R et al. ${ }^{13} 2-5 \%$ by Fisk and Storey; $1988^{6}$ and Riasko et al. 1994].14 However, Khuroo,12 1980 reported a high perinatal mortality (20 - $40 \%)$. Out of the $7 \mathrm{HBsAg}$ positive mothers in the present series, 4 had their babies seropositive for the same antigen (57.14\%). This is in accordance to the California study of acute hepatitis B in late pregnancy where HBsAg was detected in 12 out of 18 (67\%) of the babies. ${ }^{2}$

\section{CONCLUSIONS}

The present study projects viral hepatitis (Hepatitis A and E) as the major cause of jaundice in pregnancy. Despite prophylactic measures and active management protocol, $\mathrm{PPH}$ remains to be the most dreadful complication in pregnancy with Jaundice. Although low maternal mortality (5.55 \%) has 
been recorded in the present study, a high perinatal mortality $(27.78 \%)$ due to low birth weight, prematurity and birth asphyxia remains to be a matter of major concern and needs further evaluation.

Data sharing statement provided by the authors is available with the full text of this article at jemds.com.

Financial or other competing interests: None.

Disclosure forms provided by the authors are available with the full text of this article at jemds.com.

\section{REFERENCES}

[1] Reyes H, Simon FR. Intrahepatic cholestasis of pregnancy: an oestrogen related disease. Semin Liver Dis 1993;13(3):289-301.

[2] Dooley JS, Lok A, Burroughs AK. Sherlocks Disease of liver and biliary system. 12 $2^{\text {th }}$ edn. Wiley-Blackwell 2011.

[3] Tran TT. Hepatitis B virus in pregnancy. Clinical Liver Disease 2013;2(1):29-33.
[4] Tasmin AK, Lori BO. Liver disease in pregnancy. N Engi J Med. 1996;335(8):569-76.

[5] Tendon BN. Study of an epidemic of jaundice. Gastrojournal 1982: p. 739-75.

[6] Fisk NM, Storey GN. Study of Jaundice in pregnancy. J Obstet Gynaecol 1995;11:37-43.

[7] Lahiri BC. Jaundice in pregnancy. J Obstet Gynaecol India 1976;26:363-7.

[8] Griffen WC, Delts PV, Roddick JW. Jaundice in pregnancy. Chicago: Year Book of Medical Publication 1969: p. 1-56.

[9] Palmovic D. Acute viral Hepatitis in pregnancy. Results of a prospective study of 99 pregnant women. Am J Obstet 2003: p. 296-300.

[10] Chadha M, Sehgal A, Dhorje S. Hepatitis associated antigen in the sera of patient. Lancet 1970: p. 252-5.

[11] Khurana J, Teli MR. Prevalence of Hepatitis A infection and susceptibility. Am J Med 1981;70:252-5.

[12] Khuroo A. Fetomaternal outcome of jaundice. Am J Med 1980: p. 686-90.

[13] Reid R. Jaundice in adults. Br Med J 1976: p. 870-2.

[14] Jain RK. Management of jaundice in pregnancy. Medicine Update 2010;20:470-6. 